\title{
Features of syndrome $X$ develop in transgenic rats expressing a non-insulin responsive phosphoenolpyruvate carboxykinase gene
}

\author{
A.W. Thorburn, M.E. Baldwin, G. Rosella, J.D. Zajac, S. Fabris, S. Song, J. Proietto \\ Department of Medicine, University of Melbourne, Royal Melbourne Hospital, Parkville, Victoria, Australia
}

\begin{abstract}
Aims/hypothesis. Obesity, glucose intolerance, dyslipidaemia and hypertension are a cluster of disorders (syndrome $\mathrm{X}$ ) affecting many people. It has been hypothesised that these abnormalities are caused by insulin resistance, but definitive proof is lacking. We have developed transgenic rats in which the rate-limiting gluconeogenic enzyme, phosphoenolpyruvate carboxykinase, is non-insulin responsive. The aim of our study was to investigate whether syndrome $\mathrm{X}$ develops in these animals and if a high-fat diet interacts with this genetic defect.

Methods. Chow-fed transgenic and control rats aged1, 3,6 and 17 months and a subgroup of transgenic and control rats fed chow plus cafeteria foods for 6 months were examined for features of syndrome X.

Results. At 3 months, transgenic rats had fasting and postprandial hyperinsulinaemia, mild obesity (in abdominal and, to a lesser extent, peripheral regions) and fasting hypercholesterolaemia. Hypertriglycerid-
\end{abstract}

aemia was evident after 6 months while hyperglycaemia was apparent at 17 months. Hypertension had not developed by 17 months. The effect of a high-fat diet on insulin, glucose, body weight and body fat was more dramatic than the effect of the transgene alone while the effect of a high-fat diet on cholesterol and triglyceride was similar to the transgene. This illustrates that a high-fat diet is a potent catalyst for many abnormalities associated with syndrome $\mathrm{X}$. There was no evidence of an additive effect of the high-fat diet plus transgene.

Conclusion/interpretation. Therefore rats geneticallyengineered with a non-insulin responsive gluconeogenic enzyme develop several aspects of syndrome $\mathrm{X}$, supporting the hypothesis that insulin resistance initiates this cluster of disorders. [Diabetologia (1999) 42: 419-426]

Keywords Insulin resistance, syndrome X, phosphoenolpyruvate carboxykinase, glucose, insulin, triglycerides, cholesterol, obesity, transgenic rats.
The term 'syndrome $X$ ' describes a cluster of abnormalities which include glucose intolerance, obesity (particularly abdominal obesity), hypertension, insulin resistance, and dyslipidaemia characterised by

Received: 21 September 1998 and in final revised form: 23 November 1998

Corresponding author: A. Thorburn, Ph.D., Department of Medicine, Royal Melbourne Hospital, Parkville, Victoria, Australia 3050

Abbreviations: PEPCK, phosphoenolpyruvate carboxykinase (gene); NEFA, non-esterified fatty acids; MAP, mean arterial pressure; DEXA, dual energy x-ray absorptiometry raised and potentially atherogenic concentrations of low-density lipoprotein particles, diminished highdensity lipoprotein concentrations and a raised plasma triglyceride concentration [1, 2]. Clinically, syndrome $\mathrm{X}$ is also associated with increased plasma uric acid and plasminogen activator inhibitor-1 concentrations, and more recently it has been associated with defects in the innate immune system [3]. People with syndrome $\mathrm{X}$ are at risk of developing Type II diabetes and atherosclerotic cardiovascular disease [4]. These disorders are major health problems in westernized societies. Approximately $13 \%$ of persons over 55 years of age have Type II diabetes [5] and thus a three-fold higher risk of cardiovascular mortal- 
ity compared with nondiabetic subjects [6]. It is of paramount importance to identify the initial defects which could lead to syndrome $X$ and to understand its pathogenesis, if the incidence of the syndrome is to be reduced.

There is evidence that one or more genes are shared between various components of syndrome X, suggesting that a gene(s) shared between these traits is responsible for their close clustering [7, 8]. Insulin resistance is known to be an early, major feature of Type II diabetes, raising the possibility that it is the primary defect in syndrome $X$. Several population studies have identified insulin resistance as a pre-eminent feature of syndrome $X[4,9,10]$ with the result that it is often named the insulin-resistance syndrome. There are numerous mechanisms by which insulin resistance and the resulting compensatory hyperinsulinaemia could lead to the disorders of syndrome X. For example, impaired glucose tolerance could result from hyperinsulinaemia-induced betacell exhaustion [11] and obesity could result from insulin-stimulated lipogenesis or the diversion of glucose utilisation from muscle to adipocytes by hyperinsulinaemia [12]. Attempts to identify the precise role that insulin resistance has in the development of syndrome $\mathrm{X}$, however, have been confounded by interactions between insulin resistance and the environment [13-16] and interactions between insulin resistance and the other metabolic abnormalities associated with syndrome $\mathrm{X}$. For example, insulin resistance can arise as a consequence of impaired glucose tolerance by way of "glucotoxicity" mechanisms [17] or as a consequence of obesity. Abdominal fat undergoes rapid lipolysis [18] releasing high concentrations of non-esterified fatty acids (NEFA) into the blood which might induce insulin resistance by decreasing glucose oxidation [19], directly stimulating insulin release [20] and increasing the activity of hepatic gluconeogenic enzymes [21]. Therefore, positive feedback loops exist between insulin resistance and glucose intolerance and between insulin resistance and obesity which confound our understanding of what causes syndrome $\mathrm{X}$. These feedback loops and the fact that syndrome $\mathrm{X}$ could be caused by the co-inheritance of several different defects have made it difficult to identify the precise role that insulin resistance plays in the development of syndrome X.

One way of examining the role of insulin resistance in the development of this syndrome is to investigate whether the abnormalities associated with syndrome $X$ develop in an animal model with a known genetic defect that causes insulin resistance. To do this we have used a homozygous colony of rats overexpressing a non-insulin responsive phosphoenolpyruvate carboxykinase $(P E P C K)$ gene which had been developed in our laboratory [22]. $P E P C K$ catalyses the conversion of oxaloacetate to phosphoenolpyruvate in the gluconeogenic pathway. This has been shown to be a rate-limiting step in gluconeogenesis and is acutely regulated by insulin. By replacing the entire upstream-regulatory region with a metallothionein promoter, insulin cannot regulate the transcription of the enzyme, rendering the gluconeogenic pathway insulin-resistant in the transgenic rats [23]. The aims of our study were to investigate if syndrome $\mathrm{X}$ develops in these transgenic rats and to determine if an environmental factor, a high-fat 'cafeteria' diet, affects the development of this syndrome.

\section{Materials and methods}

Experimental design. Male and female transgenic Hooded Wistar rats homozygous for a non-insulin responsive, metallothionein-driven PEPCK gene and control Hooded Wistar rats derived from a heterozygous mating pair were randomly allocated into groups that were studied at weaning (1 month), 3 months, 6 months and 17 months of age. The integrity of the colony of rats was ensured by frequent testing for the transgene. We have shown that $P E P C K$ activity in these transgenic rats is increased by $14-20 \%$ in the liver and kidney [22]. We have also shown that these rats have higher endogenous glucose production in response to insulin compared with control rats and therefore are insulin-resistant [23]. Control and transgenic rats were fed mouse breeder pellets (chow) containing $20 \%$ protein, $77 \%$ carbohydrate and $3 \%$ fat (Barostock, Ridly Agriproduct Pty Ltd, Pakenham, Vic, Australia). A group of control and transgenic rats were also fed chow plus a varied selection of human palatable foods as part of a high-fat 'cafeteria' diet for 6 months. Foods offered included potato chips, biscuits, confectionery, chocolate and processed meats. In our experience a varied cafeteria diet produces much greater weight gain than a standardised high-fat diet. Chow, cafeteria foods and water were available ad libitum. Rats were housed 2-4 in a cage in a controlled environment of $20^{\circ} \mathrm{C}$ and exposed to a 12-h light-dark cycle with lights on between 0600 and 1800 hours.

Blood collection. Fasted blood was collected after a food deprivation period of 14-15 h. Post-prandial blood was collected in the morning following overnight feeding (this was not collected in the 17-month-old rats). Tail blood was collected into EDTA coated tubes placed on ice to prevent clotting. Onemonth-old rats were lightly anaesthetised by inhalation of methoxyflurane anaesthetic (Penthrane, Abbot Laboratories, North Chicago, USA) and restrained using a mouse restraint. Older rats were restrained by wrapping in a towel. At least $24 \mathrm{~h}$ recovery was allowed between the two blood collections and a volume no greater than $0.5 \%$ body weight $(5 \mu \mathrm{l} / \mathrm{g}$ body weight) was taken.

Measurement of blood parameters. Plasma glucose was measured by a glucose oxidase assay using an automated glucose analyser (Yellow Springs Instruments, Yellow Springs, Ohio, USA). Plasma insulin was determined using a double antibody radioimmunoassay (Pharmacia Diagnostics, Uppsala, Sweden). Total plasma cholesterol and triglycerides were determined using an automated spectrophotometric analyser (BM/ Hitachi System 705, Hitachi Ltd., Tokyo, Japan) using assay kits (Boehringer Mannheim GmbH Diagnostica, Munich, Germany) employing a cholesterol oxidase method [24] and a colorimetric triglyceride assay [25]. 
Measurement of blood pressure in conscious rats. Blood pressure was measured using two different methods in 6-monthold conscious rats.

Tail cuff measurements: Rats were placed under a heating lamp for approximately 5-10 min then loosely restrained by wrapping in a towel with their tail exposed. A tail cuff (15 mm diameter) was placed over the base of the rats' tail, close to the rats' body. The cuff was inflated approximately $20 \mathrm{~mm} \mathrm{Hg}$ above mean arterial pressure (MAP) and slowly deflated at a constant rate. The cuff was inflated and deflated in this manner $\sim 15$ times, upon which time MAP gradually fell to a constant level as the rats relaxed. The tail cuff was connected to a sphygmomanometer and Maclab chart recorder. Pulse and cuff pressure were recorded and the pressure at which the pulse was first detected was recorded as the MAP. The five lowest recorded measures of MAP were used to calculate the average for each rat.

Measurement of intra-arterial blood pressure using a carotid artery catheter: Rats were anaesthetised with pentobarbitone sodium (Nembutal, Boehringer Ingelheim, New South Wales, Australia) given by intraperitoneal injection $(60 \mathrm{mg} / \mathrm{kg}$ body weight) and a catheter was placed in the left carotid artery. The catheter was externalised to a dorsal region of the rat's neck and kept patent with heparinised saline $(10 \mathrm{U} / \mathrm{ml})$. Measurements of intra-arterial blood pressure were made $24-48 \mathrm{~h}$ after the catheterisation with rats being conscious and without restraint in their own cage. A pressure transducer was used to measure MAP over 2-5 min.

Body composition measurements. Total fat mass of body and abdominal region was determined in a subgroup of 6-monthold female rats by dual energy X-ray absorptiometry (DEXA) using a Hologic QDR-2000W densitometer with Hologic software version 5.67 (Hologic Inc, Waltham, Mass., USA). Rats were lightly anaesthetised with Nembutal (55 mg/ $\mathrm{kg}$ body weight). Analysis of abdominal fat was done by the same person to eliminate variation in landmarks used to identify the abdominal region.

Measurement of fat deposits. Six-month-old rats were fasted overnight, given an overdose of Nembutal by intraperitoneal injection, and immediately following death three white adipose depots (gonadal, infrarenal, and inguinal subcutaneous) were dissected from the left side of the rats and weighed.

Statistical analysis. Comparisons between variables measured in transgenic and control rats measured at more than one time point were made using univariate ANOVA with a general linear model (SPSS for Windows 8.0). Single comparisons between transgenic and control rats were done using Student's two-tailed $t$-tests. The study protocol was approved by the Royal Melbourne Hospital's Animal Ethics Committee.

\section{Results}

With the exception of body and fat weights, data for both sexes were pooled and expressed as means \pm SEM.

Plasma insulin concentrations. Fasting plasma insulin concentrations in transgenic and control rats were measured at 1,3,6 and 17 months of age (Fig. 1). ANO-

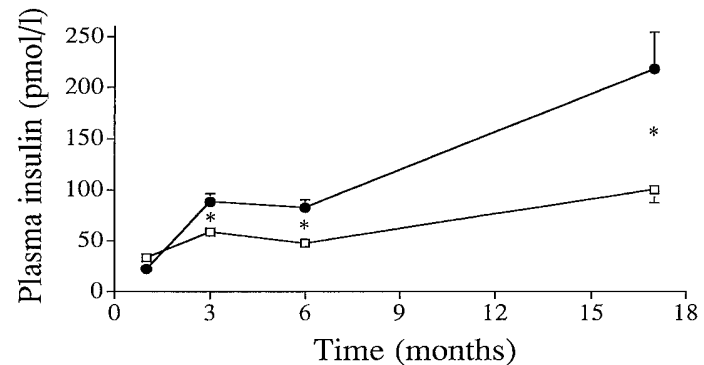

Fig. 1. Fasting plasma insulin in control and transgenic rats from weaning to 17 months of age. Results are expressed as means \pm SEM with $n=5-22$ in each group (some SEM values are too small to be seen). Asterisks indicate significant differences between control and transgenic rats $(p<0.001$ at 3 months, $p<0.01$ at 6 months, $p<0.05$ at 17 months). $-\square$, control rats; $-\longrightarrow$, transgenic rats

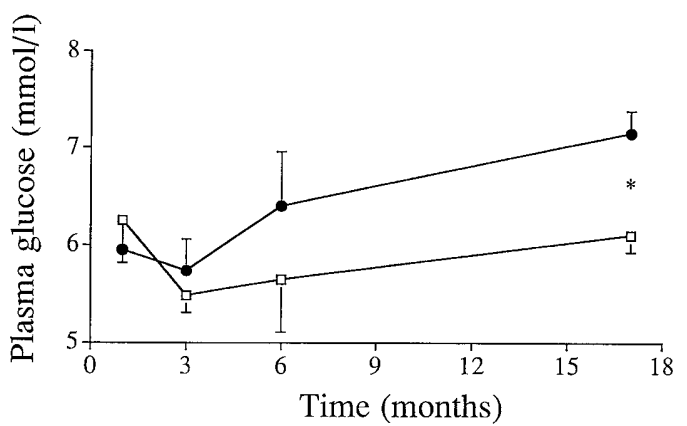

Fig. 2. Fasting plasma glucose in control and transgenic rats from weaning to 17 months of age. Results are expressed as means \pm SEM with $n=8-23$ in each group. The asterisk indicates a significant difference between control and transgenic rats $(p<0.01) .-\square-$, control rats; $-\bullet$, transgenic rats

VA showed a significant increase in insulin as animals aged in both groups $(p<0.001)$ and a significant difference between transgenic and control rats $(p<0.001)$. Fasting plasma insulin concentrations were not significantly different between transgenic and control rats at 1 month of age. Fasting plasma insulin concentrations in transgenic rats, however, were $30 \mathrm{pmol} / 1$ higher than in control rats at 3 months $(p<0.001, n=19-22)$, and this increase was maintained in transgenic rats aged 6 months. By 17 months insulin concentrations in transgenic rats were twofold higher than aged-matched control rats $(p<0.05, n=5)$.

A significant elevation in plasma insulin concentrations in transgenic rats was also observed postprandially. At 3 months, transgenic rats in the fed state had a mean plasma insulin concentration of $253 \pm 43$ compared with $119 \pm 5 \mathrm{pmol} / \mathrm{l}$ in control rats $(p<0.001)$. Post-prandial plasma insulin concentrations were also raised in transgenic rats at 6 months of age $(189 \pm 20$ vs $100 \pm 20 \mathrm{pmol} / 1, p<0.01, n=8-9)$.

Plasma glucose concentrations. Fasting plasma glucose concentrations in transgenic and control rats 
were measured at $1,3,6$, and 17 months of age (Fig. 2). No statistically significant differences in glucose concentrations were observed between transgenic and control rats at 1,3 , and 6 months of age. By 17 months of age, transgenic rats were mildly hyperglycaemic relative to control rats in the fasted state which was significant when using a $t$-test $(p<$ $0.01, n=8)$. In accordance with measures in the fasted state, post-prandial glucose concentrations were not significantly different between control and transgenic rats at 1, 3 or 6 months of age (data not shown).

Body weights. Figure 3 shows body weight growth curves for male and female control and transgenic rats from 1 to 17 months of age. ANOVA showed a significant difference in body weight between transgenic and control rats $(p<0.001)$. Body weight was not significantly different in control and transgenic rats of either sex at 1 month of age but by 3 months of age, male and female transgenic rats were significantly heavier than sex-matched control rats, and this elevated body weight relative to control rats was maintained in male and female rats aged 6 and 17 months. At 17 months of age, female transgenic rats were $30 \mathrm{~g}$ heavier than female control rats $(p<0.05, n=9-10)$ and male transgenic rats were 85 $\mathrm{g}$ heavier than male control rats $(p<0.001, n=9)$.

Fat pad weights and DEXA analysis. We measured the weights of gonadal, infrarenal and subcutaneous fat depots in female (Fig. 4A) and male (Fig. 4B) 6month-old control and transgenic rats. In female transgenic rats, the weight of periovarian and infrarenal fat pads was almost twice that recorded in control rats $(p<0.001$ for periovarian, $p<0.001$ for infrarenal, $n=10-16)$. There was a trend for subcutaneous fat to be heavier in female transgenic rats compared with control rats but this just failed to reach statistical significance $(p=0.056, n=10-16)$. Male transgenic rats had significantly heavier fat depots in the gonadal region $(p<0.01)$, the infrarenal region $(p<0.05)$ and the subcutaneous region $(p<0.01, n=8-13)$ compared with male control rats. For both male and female transgenic rats the percentage increase in fat in the abdominal gonadal and infrarenal regions was much higher than that in the peripheral subcutaneous region.

In agreement with the fat pad data, DEXA scans showed that total body fat was $54 \%$ higher in female transgenic rats compared with female control rats $(39.1 \pm 4.2$ vs $25.5 \pm 1.9 \mathrm{~g}, p<0.05, n=4-5)$. This additional fat was distributed in both the abdominal and peripheral regions. Abdominal fat (expressed as a percentage of body weight) was $43 \%$ higher in female transgenic rats compared with control rats $(17.1 \pm 1.5$ vs $12.0 \pm 0.9 \%, p<0.05)$, while peripheral fat was $34 \%$ higher in female transgenic rats compared with control rats $(11.1 \pm 0.9$ vs $8.3 \pm 0.7 \%, p<0.05)$.

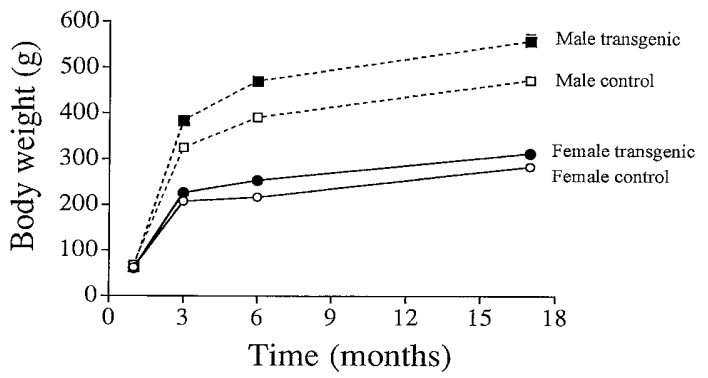

Fig. 3. Male and female body weight growth curves for control and transgenic rats recorded from weaning to 17 months of age. Results are expressed as means \pm SEM with $n=7-37$ in each group (some SEM values are too small to be seen). Significant differences between control and transgenic rats were found at 3 months ( $p<0.01$ for females, $p<0.001$ for males), 6 months $(p<0.001$ for females, $p<0.01$ for males), and 17 months ( $p<0.05$ for females, $p<0.001$ for males)

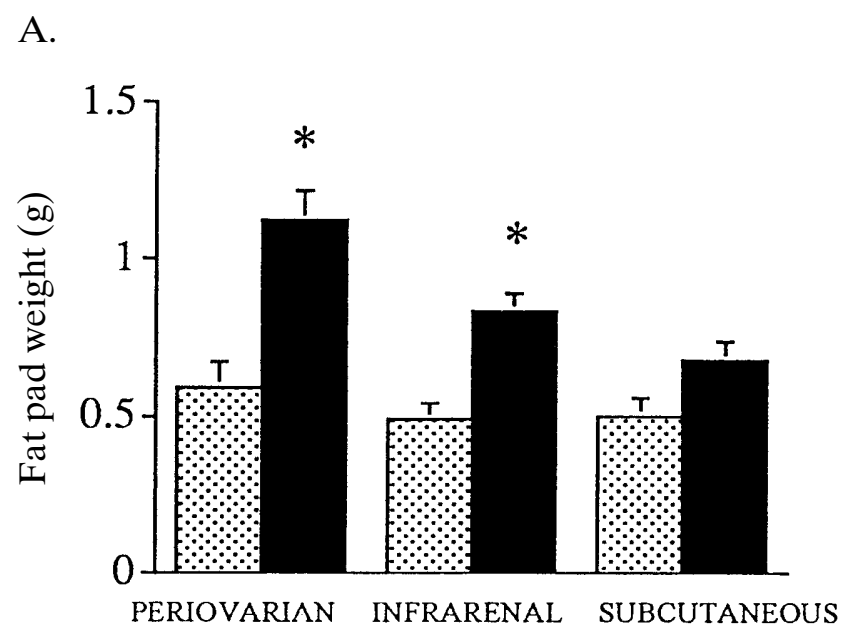

B.

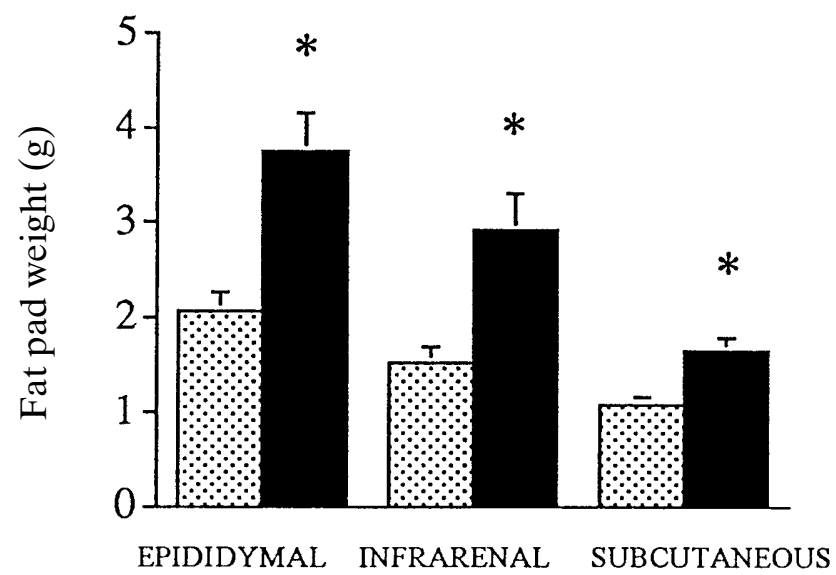

Fig. 4. A, B Weights of female (A) and male (B) white adipose tissue (gonadal, infrarenal and subcutaneous depots) in 6month-old control and transgenic rats. Results are expressed as means \pm SEM with $n=8-16$ in each group. Asterisks indicate significant differences between control and transgenic rats (for gonadal fat $p<0.001$ for females and $p<0.01$ for males, for infrarenal fat $p<0.001$ for females and $p<0.05$ for

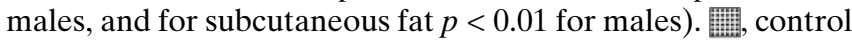
rats; $\mathbf{\square}$, transgenic rats 
Table 1. Effect of cafeteria food on fasting plasma insulin and glucose, body weight, fat pad weight, fasting plasma cholesterol and triglyceride, and blood pressure in 6-month-old control and transgenic rats. Data are presented as means \pm SEM $(n>6)$. Body and fat pad weights are presented for female rats only. ${ }^{1}$ at least $p<0.05$ compared with control rats fed the same diet. ${ }^{2}$ at least $p<0.05$ compared with chow-fed rats with the same genetic background

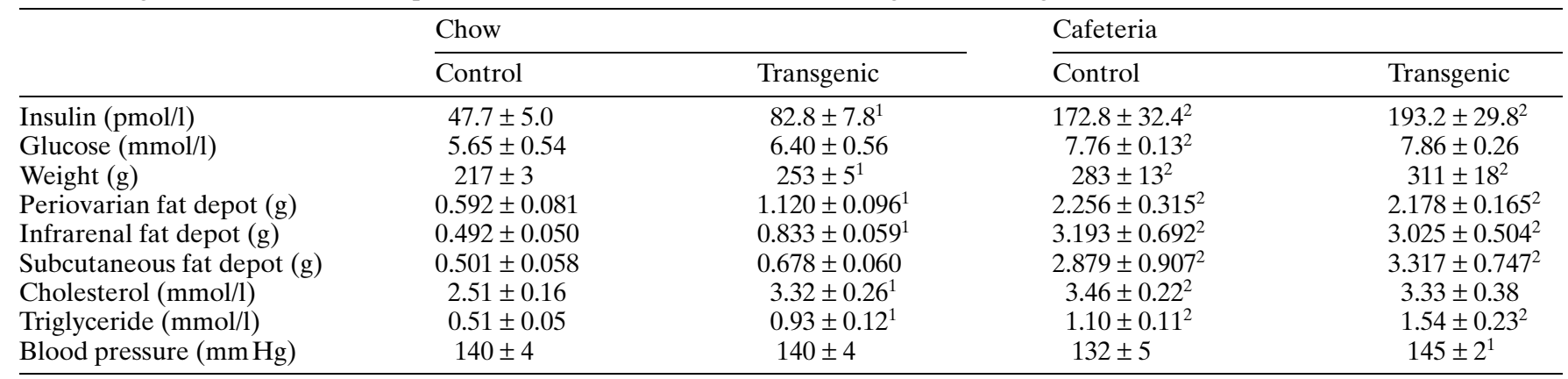

Plasma total cholesterol concentrations. Plasma total cholesterol concentrations were measured in the fasted state at 3 and 6 months (Fig. 5). Significant differences in cholesterol concentrations were noted between transgenic and control rats $(p<0.001$, ANOVA). At 3 months of age, plasma cholesterol was $42 \%$ higher in transgenic rats compared with control rats $(p<0.00001, n=14)$. This increase in plasma cholesterol was maintained in 6-month-old transgenic rats $(p<0.05, n=15)$.

Plasma triglyceride concentrations. Mean fasting triglyceride concentrations in control and transgenic rats were measured at 3 and 6 months of age (Fig. 6). ANOVA showed significant differences between control and transgenic rats $(p=0.001)$. Triglyceride concentrations were not significantly different between control and transgenic rats at 3 months of age but by 6 months of age, fasting plasma triglyceride concentrations were $82 \%$ higher in transgenic rats compared with control rats $(p<0.05, n=15)$.

Blood pressure. Blood pressure values recorded using the tail cuff and carotid cannula techniques were the same, so recordings from the two methods were pooled in the final analysis. The presence of the transgene did not affect blood pressure with both control and transgenic rats recording $140 \pm 4 \mathrm{mmHg}(n=6$ and $n=7$, respectively).

Effect of eating cafeteria food. The effect of cafeteria food on blood variables, body weight and body fat depot weights in 6-month-old rats is given in Table 1. Control and transgenic rats fed a cafeteria diet were hyperinsulinaemic compared with chow fed rats with the same genetic background (Table 1). Cafeteria fed transgenic rats did not have raised plasma insulin concentrations compared with cafeteria fed control rats, suggesting that cafeteria food did not exacerbate the hyperinsulinaemia associated with the transgene. Cafeteria food considerably raised plasma glucose

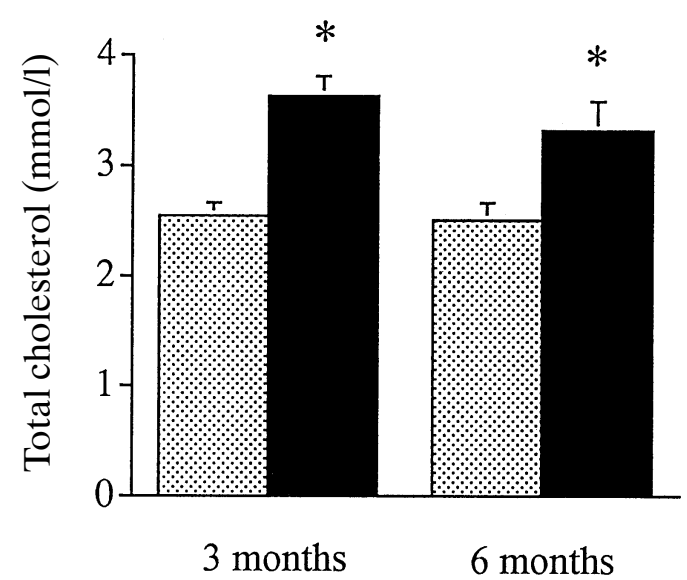

Fig. 5. Fasting plasma total cholesterol in control and transgenic rats at 3 and 6 months of age. Results are expressed as means \pm SEM $(n=14-15)$. Asterisks indicate significant differences between control and transgenic rats $(p<0.00001$

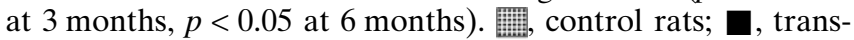
genic rats

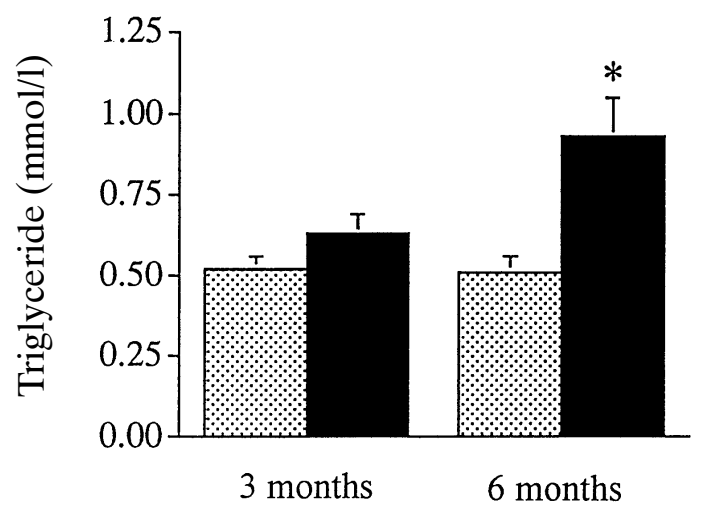

Fig. 6. Fasting plasma triglyceride in control and transgenic rats at 3 and 6 months of age. Results are expressed as means \pm $\operatorname{SEM}(n=13-15)$. A significant difference was found between control and transgenic rats at 6 months $(p<0.05)$. 啡, control rats; $\square$, transgenic rats 
concentrations in control rats but did not measurably raise glucose concentrations in transgenic rats, due to a moderate but insignificant increase in plasma glucose concentration in the chow fed transgenic rats compared with chow fed control rats (Table 1 and Fig. 2).

Eating cafeteria food increased the control rats, body weight by $30 \%$ whereas the effect of the transgene alone on body weight was more mild (17\% increase). Cafeteria food produced a similar increase in the weight of transgenic and control rats and fat pad weights in the transgenic rats were also not higher than in the control rats, indicating no cumulative effect of cafeteria feeding and insulin resistance on body weight or body fat.

Plasma cholesterol concentrations were elevated in chow fed transgenic rats compared with chow fed control rats fed the same diet (Fig. 5 and Table 1). Control rats fed a cafeteria diet also had higher plasma cholesterol concentrations than chow-fed control rats. Transgenic rats did not experience any further increase in plasma cholesterol due to cafeteria feeding. Plasma triglyceride concentrations in transgenic rats were much higher in chow fed transgenic rats than in control rats at 6 months (Fig. 6 and Table 1). The cafeteria diet produced a considerable increase in the plasma triglyceride concentrations of both control and transgenic rats when compared with chow fed control and transgenic rats respectively. There was a trend for triglyceride concentrations in transgenic rats fed a cafeteria diet to be higher than cafeteria-fed control rats but this was not statistically significant $(p=0.07)$. Eating cafeteria food had no significant effect on blood pressure in control rats and in transgenic rats. When the cafeteria diet was combined with the transgene, there was a small increase in blood pressure compared with cafeteria-fed control rats but not compared with chow-fed control rats.

\section{Discussion}

We have shown that insulin resistance induced by a non-insulin responsive PEPCK gene is associated with the early development of fasting and postprandial hyperinsulinaemia, fasting hypercholesterolaemia and mild obesity, and the subsequent development of hypertriglyceridaemia and hyperglycaemia in rats. Therefore, an inability to suppress a gluconeogenic enzyme by insulin can initiate many of the disorders associated with syndrome $\mathrm{X}$ supporting the hypothesis that insulin resistance can trigger syndrome $\mathrm{X}$. Consumption of a high-fat cafeteria diet caused a more dramatic manifestation of syndrome $\mathrm{X}$ probably because high-fat diets induce more severe insulin resistance $[14,16]$. Our data were indicative of this, showing fasting insulin values three times higher than normal values and hyperglycaemia after eating cafeteria food. Others have shown that a high-fat, refined sugar diet can induce insulin resistance and hyperinsulinaemia in Fischer rats after only 2 weeks, followed by hypertriglyceridaemia at 2 months, obesity at 5 months, and hypertension at 12 months [14] illustrating, as we have, that insulin resistance independent of obesity can be the underlying cause of syndrome $\mathrm{X}$. This is also supported by studies in non-obese, insulin-resistant mice lacking insulin receptor substrate-1 (IRS-1) which develop hypertriglyceridaemia and hypertension [26]. Therefore, insulin resistance induced either by diet or a genetic abnormality can initiate syndrome $\mathrm{X}$ in animals. There are many genes which could cause insulin resistance and potentially induce syndrome $\mathrm{X}$ in humans, however, $P E P C K$ and $I R S-1$ are unlikely to be candidates since features of syndrome $X$ do not appear to be associated with mutations in these genes in humans $[27,28]$.

It was not surprising that hyperglycaemia was a feature which developed only in older PEPCK-overexpressing rats since it has been estimated that insulin resistance may precede an inability to maintain glucose homeostasis by 15 years in humans [29]. The compensatory response of the pancreas to increase insulin secretion to maintain glucose homeostasis is not without consequences. The subsequent hyperinsulinaemia has been implicated as the primary trigger for a number of the abnormalities associated with syndrome $\mathrm{X}$ including obesity, dyslipidaemia and hypertension.

It is well-known that insulin resistance can develop as a consequence of obesity [19-21]. Our study has shown that the converse can also be true, that is, that primary insulin resistance can lead to excess fat accumulation. This may be due to hyperinsulinaemia in the transgenic rats since even small increases in insulin concentrations can have a profound effect on the storage of energy and suppression of NEFA release from adipose tissue [1]. Thus the development of syndrome $\mathrm{X}$ can be expected to progress with age as the degree of insulin resistance and hyperinsulinaemia worsens and fat depots increase in size, causing a positive feedback loop. The amount of fat located in the abdominal region appears to be important in the development of syndrome $X$ since abdominal fat is a greater risk factor for the development of insulin resistance, cardiovascular disease and Type II diabetes than total body weight $[30,31]$. The insulin-resistant, transgenic rats gained fat predominantly in the abdominal region which might have contributed to the progressive development of the syndrome in these rats.

Hyperinsulinaemia is possibly also responsible for the abnormal lipoprotein profile associated with syndrome $\mathrm{X}$ which includes raised fasting and postprandial triglyceride concentrations. Insulin can increase VLDL production and secretion by the liver [32] 
while insulin resistance is associated with downregulation of lipoprotein lipase [33]. This, combined with excess adiposity which would provide abundant substrate for the synthesis of VLDL, would contribute to the observed raised concentrations of triglycerides in our transgenic rats. Increased VLDL turnover would increase the VLDL, intermediate-density lipoprotein (IDL) and LDL particles in the circulation. Since cholesterol can be transported between tissues combined with lipoproteins, total cholesterol concentrations might also increase in response to increases in insulin. This was observed in this study.

Hypertension is associated with insulin resistance in human studies $[1,34]$. This is possibly due to direct effects of insulin on sodium reabsorption in the kidney and the sympathetic nervous system. Insulin could also cause hypertension by acting directly or indirectly on the stimulation of growth factors that can cause hypertrophy of vessel walls, resulting in narrowing of the lumen. We did not observe an increase in blood pressure in our insulin-resistant rats. It is possible that hypertension would have developed in our rats over time. Long periods of insulin resistance and compensatory hyperinsulinaemia appear to be necessary before hypertension develops in humans [34]. Hypertension, however, has been reported in IRS-1 deficient mice which are insulin resistant but not obese at 5-8 months of age [26]. It is interesting to note that when rats are made obese, either genetically [35] or by hypothalamic damage [36], and mated with spontaneously hypertensive rats, extreme hyperinsulinaemia and glucose intolerance can result but hypertension is not exacerbated [35] and can actually be attenuated [36]. These data illustrate that obese animals do not necessarily have hypertension and that hypertension is not directly related to the level of hyperinsulinaemia (at least in the short-term).

In conclusion, rats expressing a non-insulin responsive PEPCK gene have been shown to develop some of the disorders characteristic of syndrome $\mathrm{X}$ (including excess fat accumulation, raised plasma insulin, triglyceride and cholesterol concentrations). Early in life, euglycaemia is maintained at the expense of hyperinsulinaemia but mild hyperglycaemia becomes apparent as animals age and become more insulin resistant. A high-fat diet had a more pronounced effect on initiating syndrome $\mathrm{X}$ than genetically-induced insulin resistance alone. There was no significant evidence, however, of an environmentgene interaction when a high-fat diet and genetically-induced insulin resistance were combined. Our study strongly supports the hypothesis that insulin resistance can be an initiating defect in the cluster of disorders comprising syndrome $\mathrm{X}$.

Acknowledgements. This study was funded by the National Heart Foundation of Australia and a research grant from Eva and Les Erdi. The authors wish to thank S. Kantor from the Royal Melbourne Hospital Bone Density Unit for her help with the DEXA scans, J. Davis for help with feeding rats the cafeteria diet, and L. Baker and T.E. Adams from the Centre for Animal Biotechnology, Victoria, for their help in developing the transgenic rats.

\section{References}

1. Reaven G (1988) Role of insulin resistance in human disease. Diabetes 37: 1595-1607

2. Kaplan NM (1989) The deadly quartet. Upper body obesity, glucose intolerance, hypertriglyceridemia and hypertension. Arch Intern Med 149: 1514-1520

3. Pickup JC, Mattock MB, Chusney GD, Burt D (1997) NIDDM as a disease of the innate immune system: association of acute-phase reactants and interleukin- 6 with metabolic syndrome X. Diabetologia 40: 1286-1292

4. Ferrannini E, Haffner SM, Mitchell BD, Stern MP (1991) Hyperinsulinaemia: the key feature of a cardiovascular and metabolic syndrome. Diabetologia 34: 416-422

5. Harris M, Hadden W, Knowler W, Bennett P (1987) Prevalence of diabetes and impaired glucose tolerance and plasma glucose levels in U.S. population aged 20-74 years. Diabetes 36: 523-534

6. Stamler J, Vaccaro O, Neaton JD, Wentworth D (1993) Diabetes, other risk factors and 12-yr cardiovascular mortality for men screened in the Multiple Risk Factor Intervention Trial. Diabetes Care 16: 434-444

7. Mayer EJ, Newman B, Austin MA et al. (1996) Genetic and environmental influences on insulin levels and the Insulin Resistance Syndrome: an analysis of women twins. Am J Epidemiol 143: 323-332

8. Mitchell BD, Kammerer CM, Mahaney MC et al. (1996) Genetic analysis of the IRS. Pleiotrophic effects of genes influencing insulin levels on lipoprotein and obesity measures. Arterioscler Thromb Vasc Biol 16: 281-288

9. Baillie GM, Sherer JT, Weart CW (1998) Insulin and coronary artery disease: is syndrome $\mathrm{X}$ the unifying hypothesis? Ann Pharmacother 32: 233-247

10. Salonen JT, Lakka TA, Lakka HM, Valkonen VP, Everson SA, Kaplan GA (1998) Hyperinsulinemia is associated with the incidence of hypertension and dyslipidemia in middle-aged men. Diabetes 47: 270-275

11. DeFronzo RA, Bonadonna RC, Ferrannini E (1992) Pathogenesis of NIDDM. A balanced overview. Diabetes Care 15: 318-368

12. Cusin I, Terrettaz J, Rohner-Jeanrenaud F, Jeanrenaud B (1990) Metabolic consequences of hyperinsulinemia imposed on normal rats on glucose handling by white adipose tissue, muscles and liver. Biochem J 267: 99-103

13. Ferrannini E (1992) The insulin resistance syndrome. Curr Opin Nephrol Hypertens 1: 291-298

14. Barnard RJ, Roberts CK, Varon SM, Berger JJ (1998) Diet-induced insulin resistance precedes other aspects of the metabolic syndrome. J Appl Physiol 84: 1311-1315

15. Phillips DI (1998) Birth weight and the future development of diabetes. A review of the evidence. Diabetes Care 21 [Suppl 2]: B 150-155

16. Storlien LH, Baur LA, Kriketos AD et al. (1996) Dietary fats and insulin action. Diabetologia 39: 621-631

17. McClain DA, Crook ED (1996) Hexosamines and insulin resistance. Diabetes 45: 1003-1009

18. Wahrenberg H, Lonnqvist F, Arner P (1989) Mechanisms underlying regional differences in lipolysis in human adipose tissue. J Clin Invest 84: 458-467

19. Jenkins AB, Storlien LH, Chisholm DJ, Kraegen EW (1988) Effects of nonesterified fatty acid availability on tis- 
sue-specific glucose utilization in rats in vivo. J Clin Invest 82: 293-299

20. Boden G, Chen X, Rosner J, Barton M (1995) Effects of a 48-h fat infusion on insulin secretion and glucose utilization. Diabetes 44: 1239-1242

21. Andrikopoulos S, Rosella G, Kaczmarczyk S, Zajac J, Proietto J (1996) Impaired regulation of hepatic fructose-1,6bisphosphatase in the New Zealand Obese mouse: An acquired defect. Metabolism 45: 622-626

22. Rosella G, Zajac JD, Baker L et al. (1995) Impaired glucose tolerance and increased weight gain in transgenic rats overexpressing a non-insulin responsive phosphoenolpyruvate carboxykinase gene. Mol Endocrinol 9: 1396-1404

23. Dickens RA, Rosella G, Zajac J, Proietto J (1997) Insulin inhibits gluconeogenesis predominantly through suppression of phosphoenolpyruvate carboxykinase. Diabetologia 40: A 24

24. Allain C, Poon L, Chan C, Richmond W, Fu P (1974) Enzymatic determination of total serum cholesterol. Clin Chem 20: 470-475

25. Megraw R, Dunn D, Biggs H (1979) Manual and continuous-flow colorimetry of triacylglycerols by a fully enzymic method. Clin Chem 25: 273-278

26. Abe H, Yamada N, Kamata K et al. (1998) Hypertension, hypertriglyceridemia, and impaired endothelium-dependent vascular relaxation in mice lacking insulin receptor substrate-1. J Clin Invest 101: 1784-1788

27. Ludwig DS, Vidal-Puig A, O'Brien RM et al. (1996) Examination of the phosphoenolpyruvate carboxykinase gene promoter in patients with noninsulin-dependent diabetes mellitus. J Clin Endocrinol Metab 81: 503-506
28. Ossei-Gerning N, Mansfield MW, Stickland MH, Grant PJ (1997) Insulin receptor substrate-1 gene polymorphism and cardiovascular risk in non-insulin dependent diabetes mellitus and patients undergoing coronary angiography. Clin Lab Haematol 19: 123-128

29. Sacks D, McDonald J (1996) The pathogenesis of Type II diabetes mellitus - a polygenic disease. Am J Clin Pathol 105: $149-156$

30. Bjorntorp P (1994) Abdominal obesity and the metabolic syndrome. Ann Med 24: 465-468

31. Despres JP (1993) Abdominal obesity as an important component of insulin-resistance syndrome. Nutrition 9: 452-459

32. Steiner G (1994) Hyperinsulinemia and hypertriglyceridaemia. J Intern Med 236 [Suppl 736]: 23-26

33. Maheux P, Azhar S, Kern PA, Chen YD, Reaven GM (1997) Relationship between insulin-mediated glucose disposal and regulation of plasma and adipose tissue lipoprotein lipase. Diabetologia 40: 850-858

34. DeFronzo R, Ferrannini E (1991) Insulin resistance - a multifaceted syndrome responsible for NIDDM, obesity, hypertension, dyslipidemia, and atherosclerotic cardiovascular disease. Diabetes Care 14: 173-194

35. Friedman JE, Ishizuka T, Liu S et al. (1997) Reduced insulin receptor signalling in the obese spontaneously hypertensive Koletsky rat. Am J Physiol 273: E1014-E1023

36. Iwase M, Yamamoto M, Iino K et al. (1998) Obesity induced by neonatal monosodium glutamate treatment in spontaneously hypertensive rats: an animal model of multiple risk factors. Hypertens Res 21: 1-6 